\title{
Weiterarbeit im Rentenalter: Zurückliegende Entwicklungen, aktueller Stand des Wissens und offene Fragen
}

\author{
J. Deller und L. Naegele
}

\section{Einleitung}

Dieser Beitrag blickt zunächst auf einen Kongress zum demografischen Wandel und zur Zukunft der Erwerbsarbeit im Jahr 1999 zurück und beschreibt seine Kernaussagen zur Arbeit im Rentenalter. Vor diesem Hintergrund wird die Situation des Jahres 2020 betrachtet. Dabei werden zwei bedeutsame Felder vorgestellt und diskutiert: Erstens auf der individuellen Ebene neuere empirische Erkenntnisse zur Situation arbeitender Personen im Rentenalter, zweitens auf der organisationalen Ebene empirisch geprüfte Dimensionen von Arbeit im Rentenalter, wie sie der Later Life Workplace Index beschreibt. Der Beitrag schließt mit einer Diskussion (noch) offener Fragen und möglicher Forschungsanstöße.

\section{Weiterarbeit im Rentenalter aus der Perspektive von Praxis - ein Blick zurück}

Am 29. und 30. November 1999 fand in Berlin ein Kongress mit mehr als 200 Teilnehmerinnen und Teilnehmern und internationaler Beteiligung zum Thema „Altern und Arbeit" statt. Besprochen wurden Ergebnisse des Forschungsschwerpunkts „Demographischer Wandel und die Zukunft der Erwerbsarbeit am Standort Deutschland" des Bundesministeriums für Bildung und Forschung (BMBF). Vertreten waren 20 öffentliche oder private Forschungseinrichtungen, deren Beiträge verschiedene thematische Aspekte des Forschungsschwerpunkts abbildeten. So finden sich in der Dokumentation von Rothkirch (2000) Themen wie Chancen alternder Arbeitnehmerinnen und Arbeitnehmer im Erwerbsleben, wenn neue Qualifikationen für mobile, flexible und virtuelle Arbeit erforderlich sind, welche einzel-, zwischen- und überbetriebliche Maßnahmen zur Arbeitsgestaltung und Personalentwicklung die Innovationsfähigkeit alternder Belegschaften erhalten und fördern können oder wie die Altersstruktur der Belegschaft eines Unternehmens aussehen sollte, damit die spezifischen Kompetenzen, 


\section{J. Deller und L. Naegele}

Erfahrungen und Innovationspotenziale von Mitarbeiterinnen und Mitarbeitern aller Arbeitsgruppen optimal genutzt werden.

Für das Thema des vorliegenden Beitrags ist insbesondere interessant, welche Aussagen sich im Tagungsband zur Weiterarbeit im Rentenalter finden. In der Rückschau über zwei Dekaden fallen verschiedene Aspekte auf: Erstens schienen betriebliche Vertreterinnen und Vertreter dem Konzept der Rente mit 60 verhaftet gewesen zu sein. Der Blick blieb in der gelebten betrieblichen Realität. So beschrieb Ax (2000) das Modell „Hau rein bis 55 - und genieße dann einen frühen Ruhestand“, das Betriebe präferierten. Angenommen wird bei diesem Modell das Anbrechen eines Reiches der Freiheit mit der Rente. Hier spiegelt sich die damalige Rentenund Arbeitsmarktpolitik, die - von der Politik subventioniert - Anreize zur frühen Ausgliederung setzte. Folglich hieß ein von Spieker (2000) geschildertes Projekt bei Volkswagen „Gesund arbeiten bis zur Rente“. Dabei endete die „Flexibilität in der Arbeitszeitgestaltung“ mit 60 Jahren, allerspätestens jedoch mit Erreichen des Rentenalters. Für Morschhäuser (2000, S. 283) war damals „betriebliche Personalplanung mit Blick auf ältere Beschäftigte (...) in erster Linie Planung von Personalfreisetzung." Zweitens wurde nach Böhle (2000) zudem eine weitere Reduzierung der Altersgrenze (Rente mit 60 und weniger) als keineswegs abwegig diskutiert, während Reformen in den Institutionen sozialer Sicherung, beispielsweise eine Flexibilisierung der Altersgrenze sowohl nach unten wie nach oben, die Möglichkeit zur Kombination von Sozialleistungen mit Arbeitseinkommen sowie die Verkopplung von Rechtsansprüchen auf Leistungen mit Wahlmöglichkeiten des Umfangs ihrer faktischen Inanspruchnahme lediglich im Konjunktiv angesprochen wurden. Drittens wies Kruse (2000) im Zusammenhang mit dem Übergang von Arbeit in Rente in dem damaligen Band auf gesetzliche Möglichkeiten wie die Teilrente und das Altersteilzeitmodell zum gleitenden Ausstieg aus dem Erwerbsleben hin. Eine breite Umsetzung dieser Formen der Transition in den Ruhestand sah er jedoch als gescheitert an. Viertens wurde neben dem Konzept der Rente mit 60 auch die Möglichkeit längerer Erwerbszeiten diskutiert, im Regelfall im Sinne von späteren Rentenzugängen vor Erreichen des gesetzlichen Rentenalters, jedoch nicht die konkrete Gestaltung von Arbeit in der Rente auf individueller und organisationaler Ebene. Fünftens fanden sich über den aktuellen betrieblichen Rahmen hinausgehende Ideen im Regelfall nicht bei den Praxisvertreterinnen und -vertretern, sondern bei Personen, die politiknah und in der Wissenschaft tätig sind. So legte Helfferich (2000) ihr Augenmerk darauf, dass Menschen länger arbeiten können, dass sie später in die Rente gehen und aktiver altern. Auch sollte älteren Menschen eine bessere Integration und der längere Verbleib im Arbeitsmarkt 
ermöglicht werden, um später in Rente zu gehen. Der Soziologe Böhle (2000) konnte sich auch eine zweite Ebene der Flexibilisierung vorstellen, die an dem Punkt ansetzt, an dem damals die Verrentung begann. Das vor zwei Dekaden vorherrschende Modell sei nach der Prämisse alles oder nichts konstruiert worden, entweder Erwerbsarbeit oder Rente. Böhle forderte sehr viel flexiblere Formen von Teilrente mit Teilerwerbsfähigkeit, möglicherweise auch kombiniert mit der Schaffung völlig neuer Beschäftigungsformen. Dabei dachte Böhle kreativ auch an Beschäftigungsformen jenseits von Markt und Staat, in Zwischenbereichen, die die Grundlage dafür bilden, möglichst lange in Erwerbsarbeit eingegliedert zu sein.

Sechstens baute Behrens (2000) unter Nutzung von Lebensphasenmodellen eine Brücke in die heutige Zeit. Er stellte unter Bezug auf die Ergebnisse des Verbundprojektes „Problem der begrenzten Tätigkeitsdauer und neue Arbeitszeitmodelle für ältere Arbeitnehmer - Altersintegrierte Arbeitspolitik“ (Behrens et al. 1999) die Frage, wie Erwerbsarbeit im Leben verteilt ist. Behrens betont, dass das „sozialverträgliche“ und kostengünstige Verlassen eines Betriebes leistungsgewandelter, darunter auch älterer Beschäftigter, die altersgerechte Gestaltung von Arbeitsplätzen und Einrichtung neuer beruflicher Laufbahnen verhindert. Erst wenn das Einwerben nachfolgender Personen nicht gelinge, entstehe aus einzelbetrieblicher Sicht die Notwendigkeit der altersgerechten Gestaltung von Arbeitsplätzen und der Einrichtung betrieblicher Laufbahnen. Denn diese würde es den damals ausscheidenden Kohorten ermöglichen, weiterhin im Betrieb zu verbleiben. Damit wies er bereits vor 20 Jahren auf eine Lösungsmöglichkeit der demografischen Herausforderung hin, in der größere Kohorten verrentet werden, als nachwachsen. Schon bei gleichbleibender Nachfrage nach Arbeitskräften entsteht durch die Nachbesetzungslücke die Notwendigkeit, ältere Arbeitskräfte im Betrieb zu halten und dazu Arbeitsplätze altersgerecht anzupassen und neue berufliche Laufbahnmodelle zu konzipieren und $\mathrm{zu}$ implementieren. Damals, so ein Kerngedanke Behrens (S. 235f.), umfasste das Spektrum betrieblicher Reaktionen auf arbeitsplatzspezifisch begrenzte Tätigkeitsdauer drei Strategien: Arbeitsplatzgestaltung, betriebliche und überbetriebliche Laufbahnpolitik sowie Externalisierung der betroffenen Beschäftigten aus ihren Betrieben heraus (zu anderen Betrieben, in die Arbeitslosigkeit, in die Frührente). Diese drei Möglichkeiten sah er als wechselseitig begrenzt substitutiv an. Je mehr eine von den dreien genutzt werden kann, umso weniger musste von den beiden anderen Gebrauch gemacht werden. Je „sozialverträglicher“ leistungsgewandelte, darunter auch ältere Beschäftigte zum Verlassen eines Betriebes bewegt werden und neue eingeworben werden konnten, umso weniger notwendig war aus einzelbetrieblicher Sicht die altersgerechte Gestaltung 
von Arbeitsplätzen und Einrichtung betrieblicher Laufbahnen. Auer (2000) unterstütze diese Sichtweise und argumentierte, ältere Arbeitskräfte würden dem Markt länger zur Verfügung stehen müssen, weil das Angebot an jungen, in den Markt eintretenden Personen zurückgehen wird und weil die Finanzierungsprobleme der Alterssicherungssysteme zunehmen. Diese Entwicklung begründete außerdem Zweifel an der bisher verfolgten Politik der Frühverrentung. Tatsächlich schien bereits Auer die Strategie, das Arbeitskräfteangebot durch Frühverrentungen zu reduzieren, überholt. Nicht die Verringerung des Arbeitskräfteangebotes, sondern seine Ausweitung durch längere Lebensarbeitszeiten sah er als politische Priorität. In jedem Falle erfordert der Alterungstrend ein Umdenken gegenüber älteren Arbeitskräften und aktives Handeln durch die Ausweitung von Möglichkeiten der Weiterbildung sowie produktiver und sinnvoller Arbeit auch für Ältere. Insgesamt beurteilte Kador (2000, S. 347) jedoch: „Verglichen mit dem Problemdruck ... sind die Ansatzpunkte für Lösungen eher dünn gesät.“ Es müsse darum gehen, „Strategien zur Integration Älterer in den Erwerbsprozess zu finden, zu entwickeln und weiter zu verbreiten." Dem pflichtete Naegele (2000a, 2000b) bei, indem er den deutlich erkennbaren Bedarf an älteren und zugleich qualifizierten und produktiven Arbeitskräften, insbesondere nach 2010/2015, identifizierte. Naegele (2000a, S. 437) schrieb: „Was hingegen fehlt, ist der Transfer (...) in Praxis- und Politikkonzepte, die es ermöglichen, dass künftig mit älteren und anders zusammengesetzten Belegschaften (...) produktiv und innovativ gearbeitet (...) werden kann." Naegele forderte bereits im Jahr 2000 einen Paradigmenwechsel von einer reaktiven „älteren Arbeitnehmerpolitik“ zu einer präventiven, lebenslauforientierten, ,altersneutralen“ Politik der Beschäftigungsförderung und -sicherung alternder Belegschaften. Dies ergänzte er unter anderem mit der Forderung zur Neuorganisation von Lebensarbeitszeit.

Aus heutiger Sicht schienen insbesondere zwei Sichtweisen vorzuherrschen: Betrieblich war die Argumentation an der Rente mit 60 Jahren ausgerichtet, während außerhalb der Betriebe durchaus differenzierte Konzepte zu einer anderen Gestaltung der Arbeitswelt im demografischen Wandel vorgedacht wurden. Diese neuen Konzepte haben damals jedoch kaum Eingang in die betriebliche Realität gefunden. Dies lag auch daran, dass insbesondere zur Arbeit im Rentenalter nur ansatzweise Erkenntnisse vorlagen, was sich in den Folgejahren jedoch verändert hat.

Dieser Beitrag möchte im Weiteren einen Blick auf die aktuelle Situation werfen und dabei insbesondere zwei bedeutsame Felder diskutieren: Zunächst werden auf der individuellen (Mikro-)Ebene neuere empirische Erkenntnisse zur Situation arbeitender Rentnerinnen und Rentner zusam- 
mengefasst und dann auf der organisationalen (Meso-)Ebene empirisch entwickelte und validierte Dimensionen von Arbeit im Rentenalter, wie sie der Later Life Workplace Index (LLWI) beschreibt, präsentiert. Der Beitrag schließt mit einer Diskussion (noch) offener Fragen und möglicher Forschungsanstöße ans Feld.

\section{Die individuelle Perspektive von Arbeit in der Rente - Was wissen wir heute?}

Für die Arbeit in der Rente finden sich in der einschlägigen Literatur verschiedene Begrifflichkeiten. Während es sich bei Bridge Employment um ein im nordamerikanischen Raum weitverbreitetes Phänomen handelt, lagen in Deutschland zur Arbeitsmarkteilnahme aktiver Ruheständlerinnen und Ruheständler keine Zahlen vor. Erste grundlegende empirische Ergebnisse für Deutschland, zunächst unter dem Begriff des Silver Work, berichten u. a. Deller, Liedtke und Maxin (2009), Deller und Maxin (2009) sowie Maxin und Deller (2010). Resultate dieser explorativen Studie und weiterer Beiträge nutzte eine Arbeitsgruppe in Koordination durch das Bundesinstitut für Bevölkerungsforschung für eine erste bevölkerungsrepräsentative TOP-Studie („Transition and Old Age Potential“) in Deutschland im Jahr 2013. Befragt wurden in einer ersten Welle insgesamt 5.002 zufällig ausgewählte Personen im Alter zwischen 55 und 70 Jahren. Der Schwerpunkt der Fragen lag auf dem Übergang in den Ruhestand. In den Jahren 2015/2016 folgte die zweite Welle mit der erneuten Befragung der Personen der ersten Welle, diesmal mit dem Schwerpunkt Umsetzung von früheren Plänen beim Übergang in den Ruhestand. Schließlich wurden in der dritten Welle die Personen der zweiten Welle und ihrer Partnerinnen und Partner befragt. Seither liegen belastbare und aussagefähige Daten vor. Einzelne Ergebnisse aus den Daten der TOP-Studie sollen hier geschildert werden (Büsch, Zohr, Brusch, Deller, Schermuly, Stamov-Roßnagel und Wöhrmann 2015; Fasbender, Deller, Zohr, Büsch, Schermuly und Mergenthaler 2015; Hess und Naegele, im Erscheinen; Mergenthaler, Konzelmann, Cihlar, Micheel und Schneider 2020; Naegele und Hess 2018; Naegele, Stiemke, Hess und Mäcken 2020).

Zunächst gehen wir auf Absichten ein, im Ruhestand tätig zu sein. Fasbender et al. (2015) beschäftigen sich mit individuellen Perspektiven, während Büsch et al. (2015) vier Typen von Absichten der Weiterbeschäftigung im Ruhestand identifizieren. Naegele et al. (2020) sowie Naegele und Hess (2018; im Erscheinen) thematisieren im Weiteren dann konkrete Ausgestaltungswünsche einer Erwerbstätigkeit im Ruhestand, während der Beitrag von Mergenthaler et. al (2020) generelle Entwicklungen sowie 
Merkmale einer tatsächlichen Beschäftigung im Rentenalter zwischen 2013 bis 2019 nachzeichnet. ${ }^{1}$

Fasbender et al. (2015) überprüften zentrale Einflussfaktoren auf die $A b$ sicht erwerbstätiger und nichterwerbstätiger 55-70-Jähriger, im zukünftigen Ruhestand tätig zu sein. Untersucht wurden soziodemografische, familiale, individuelle und arbeitsbezogene Faktoren. Zunächst halten Fasbender et al. (2015) fest, dass die Absicht zur Erwerbstätigkeit im Ruhestand bei erwerbstätigen Personen deutlich höher ist als bei den Nichterwerbstätigen. Die weitere Analyse belegt, dass bei den Erwerbstätigen bevölkerungsrepräsentativ jede dritte Person (36\%) eine grundsätzliche Absicht zur Weiterbeschäftigung im Ruhestand angibt. Über alle Alterskategorien hinweg haben Männer eine höhere Erwerbsabsicht als Frauen. Interessanterweise ist bei beiden Geschlechtern ein Gradient der Erwerbsabsicht hinsichtlich der Gemeindegröße zu konstatieren: Je kleiner die Gemeinde, desto geringer die Absicht zur Erwerbstätigkeit im Ruhestand. Während sich in Gemeinden unter 10.000 Einwohnern nur ein Drittel der Frauen (32\%) und 41\% der Männer grundsätzlich eine Erwerbstätigkeit im Ruhestand vorstellen können, sind dies in größeren Städten mit mehr als 100.000 Einwohnern 40\% der Frauen und sogar jeder zweite Mann (51\%). Dabei zeigen sich allerdings klare Unterschiede im Bildungs- und Gesundheitsgrad, so dass eine hohe Ausprägung in diesen beiden Bereichen jeweils mit einer höher ausgeprägten Weiterbeschäftigungsabsicht einhergeht. Demnach sind es die Gesunden und die hoch sowie gering Ausgebildeten (nicht jedoch Personen mit mittlerer Ausbildung), die planen, im Ruhestand weiterhin beruflich tätig zu sein. Als weiteren Einfluss berichtet die Autorengruppe die subjektiv eingeschätzte finanzielle Lage: Es beabsichtigen eher diejenigen im Ruhestand weiterzuarbeiten, die ihre eigene finanzielle Lage als schlechter einschätzen. Höhere Absichtserklärungen können ebenfalls bei Menschen mit Migrationshintergrund und bei Selbstständigen erkannt werden. Außerdem bestehen tendenzielle Einflüsse der Persönlichkeit. So sind Verträglichkeit und Gewissenhaftigkeit negativ mit der ruhestandsbezogenen Erwerbsabsicht verknüpft. Extraversion hingegen weist einen positiven Zusammenhang zur Absicht zur Erwerbstätigkeit im Ruhestand auf. Fasbender et al. (2015) machen deutlich, wie individuell unterschiedlich sich Weiterbeschäftigungsabsichten darstellen. Zudem zeigen sie auf, wie bedeutsam es für die Gestaltung der Beschäftigung von älteren Arbeitnehmerinnen und Arbeitnehmern sein wird, differenziert auf einzelne Personen einzugehen und dementsprechend Erwerbstätigkeit im Ruhestand individuell im Dialog zu gestalten.

Büsch et al. (2015) analysieren Muster von Absichten zur Weiterbeschäftigung im Ruhestand. Mithilfe von Clusteranalysen identifizieren sie Grup- 
pen, die auf der Neigung zur Erwerbstätigkeit im Ruhestand, der gewünschten Arbeitszeit und dem Wunsch beim Arbeitgeber zu bleiben basieren. Vier distinkte Typen sind abgrenzbar: Die Entschlossenen, die Wechsler, die Stetigen und die Ambitionierten. Bei ihnen bestehen Unterschiede in soziodemografischen, arbeitsbezogenen und familialen Variablen. Diese zeigen auf, dass sich insbesondere die Ambitionierten (hohe Neigung zur Weiterbeschäftigung und hohe gewünschte Arbeitszeit) von den anderen drei Gruppen in Geschlecht, Alter, Offenheit, momentaner Arbeitszeit, beruflicher Stellung und Anzahl der Mitarbeiterinnen und Mitarbeiter unterscheiden. Diese Gruppe beinhaltet verstärkt Männer, am häufigsten Selbstständige und Personen, die in Kleinstbetrieben unter zehn Mitarbeiterinnen und Mitarbeitern tätig sind. Die Weiterbeschäftigungswilligen stellen insgesamt eine stetig wachsende Gruppe dar: Sie ist aufgrund der erkannten Unterschiede in den Mustern von Wünschen und Absichten zur Weiterbeschäftigung im Ruhestand zudem als heterogen anzusehen. Flexible Gestaltungsmöglichkeiten von Arbeitszeiten und -inhalten sowie typgerechte Weiterbeschäftigungsangebote können demnach Möglichkeiten zur zukünftigen Reaktion des Arbeitsmarktes auf die erhöhte Beschäftigungsquote älterer Menschen sein.

Naegele und Hess (2018), Naegele et al. (2020) sowie Hess und Naegele (im Erscheinen) gehen der gewünschten konkreten Ausgestaltung einer Erwerbstätigkeit im Ruhestand nach. Bezogen auf die zeitliche Ausgestaltung zeigt sich, dass die Befragten im Durchschnitt ca. 17 Std. an zwei Tagen in der Woche im Ruhestand arbeiten wollen. Mit Blick auf Geschlecht und Bildung zeigen sich bei Anzahl der Tage, die sich Personen wünschen zu arbeiten, kaum Unterschiede, jedoch wollen Männer, Hochqualifizierte und Selbstständige tendenziell mehr Stunden arbeiten als Frauen und Niedrigqualifizierte (Naegele et al. 2020). Blickt man auf die Beschäftigungsverhältnisse, zeigt sich, dass etwa ein Drittel der Befragten im Rahmen einer Selbstständigkeit im Ruhestand arbeiten wollen, hiervon allerdings ein nicht unerheblicher Teil beim ehemaligen Arbeitgeber und es finden sich Geschlechter- und Bildungsunterschiede. Lediglich jede vierte Frau und nur ca. 15\% der Niedrigqualifizierten möchten selbstständig beschäftigt sein, während der Anteil bei Männern und Hochqualifizierten deutlich höher liegt (Naegele und Hess 2018). Lohnenswert ist auch ein Blick auf die gewünschten konkreten Arbeitsinhalte bzw. die Frage, ob Personen sich wünschen, der „gleichen“ oder „einer anderen“ Tätigkeit im Ruhestand nachzugehen. Hier zeigt sich, dass sich zwar die überwältigende Mehrheit wünscht, die gleiche Tätigkeit wie vor dem Ruhestand auszuüben, sich jedoch etwa ein Viertel der Befragten eine neue Tätigkeit mit anderen Arbeitsinhalten und z.T. auch bei einem anderen Arbeitgeber im 
Ruhestand wünscht. Insbesondere dieser Gruppe, so das Fazit der Autorinnen und Autoren, gilt es neue Karriereentwicklungsperspektiven - auch im Ruhestand - zu eröffnen.

Fragt man nach der tatsächlichen Umsetzung dieser formulierten Wunschvorstellungen zeigen Mergenthaler et al. (2020), dass die Zabl der Erwerbstätigen im Rentenalter (ab 65 Jahre) sich seit dem Jahr 1998 mehr als verdreifacht hat. Nach Mergenthaler et al. (2020) stiegen diese Zahlen auf 790.000 (Männer) und 495.000 (Frauen) im Jahr 2018. Die TOP-Daten zeigen für das Jahr 2019 einen Anteil der Erwerbstätigen bei den Männern von 23 Prozent und bei den Frauen von 10 Prozent. Arbeit im Rentenalter ist offensichtlich nicht selten. Der Anteil der Selbstständigen unter den weiterhin am Arbeitsmarkt aktiven Altersrentnerinnen und Altersrentner liegt im Jahr 2019 bei 28 Prozent. Im Rentenalter weiterarbeitende Frauen sind häufiger als Männer abhängig beschäftigt. Zudem wechseln sie seltener den Arbeitgeber. Dagegen zeichnen sich männliche Erwerbsverläufe im Rentenalter häufiger durch Arbeitgeberwechsel oder den Übergang in die berufliche Selbstständigkeit aus. Dies deuten Mergenthaler et al. (2020) als Ausdruck einer günstigeren Arbeitsmarktposition der männlichen Befragten. Mit Blick auf die Arbeitszeit sind 94 Prozent der Befragten in Teilzeit, also weniger als 30 Stunden wöchentlich, aktiv. Eine fortgeführte Tätigkeit in Teilzeit kann als überbrückender gleitender Übergang in den Ruhestand verstanden werden. Die TOP-Studie berichtet einen Einkommensmedian aus einer Erwerbstätigkeit im Rentenalter bei Männern von 400 Euro pro Monat. Frauen verdienen im Median mit 225 Euro im Jahr 2019 deutlich weniger. Geringfügige Beschäftigungen bzw. 450-Euro-Jobs sind im Rentenalter häufig zu beobachten. Im Jahr 2016 betrug der Anteil geringfügig Beschäftigter unter allen Erwerbstätigen im Rentenalter 71 Prozent (2019: Männer: 69 Prozent, Frauen 93 Prozent). Für Frauen ist die geringfügige Beschäftigung demnach der Normalfall.

Welche Merkmale haben Beschäftigte im Rentenalter? Körperliche Gesundheit begünstigt Erwerbstätigkeit. So sind zum Zeitpunkt der Erstbefragung 38 Prozent der Personen, deren körperliche Gesundheit im oberen Drittel der Stichprobe liegt, erwerbstätig, während es bei Befragten im unteren Drittel nur 23 Prozent sind. Die Erwerbsbeteiligung im Rentenalter ist bei mittleren Bildungsgruppen am höchsten. Bei Befragten mit mittlerer bzw. hoher Bildungsdauer übte jede dritte Person im Rentenalter noch eine bezahlte Tätigkeit aus. Bei niedrigen Bildungsabschlüssen waren dies 25 Prozent. Mergenthaler et al. (2020) vermuten, dass dies bei Menschen mit geringer Bildungsdauer unter anderem auf ungünstige Chancen am Arbeitsmarkt und einer im Allgemeinen schlechteren Gesundheit zurückgeführt wird. Bei Personen mit hoher Bildungsdauer spielen ihrer Interpretation 
nach fehlende Notwendigkeiten zu einer fortgeführten Erwerbstätigkeit aufgrund einer günstigen materiellen oder gesundheitlichen Situation sowie konkurrierende Lebens- bzw. Konsumziele eine Rolle. Die Möglichkeit der Weiterbeschäftigung beim letzten Arbeitgeber begünstigt eine Erwerbstätigkeit im Ruhestand. Gleichzeitig spielt die berufliche Zufriedenheit nach den vorliegenden Ergebnissen für die Dauerhaftigkeit einer Erwerbstätigkeit im Rentenalter eine wichtige Rolle. In Bezug auf die Motive einer Erwerbstätigkeit werden am häufigsten soziale und materielle Erwerbsmotive genannt. Bei den Männern wird im Jahr 2016 am häufigsten „Kontakt zu anderen Menschen" genannt, dicht gefolgt von dem finanziellen Motiv, „weiterhin Geld verdienen“ zu wollen. „Spaß an der Arbeit“, „noch eine Herausforderung haben“ und das „Gefühl, gebraucht zu werden“ folgen auf den weiteren Rängen. Knapp die Hälfte der Frauen nennt im Jahre 2016 „Spaß an der Arbeit“ als Hauptmotiv. Wie auch bei den Männern wird „weiterhin Geld verdienen“, also das finanzielle Motiv, am zweithäufigsten als Erwerbsgrund genannt. Im Unterschied zu den Männern wird „Kontakt zu anderen Menschen“ als Motiv von den Frauen nur am dritthäufigsten genannt. Es folgen wie auch bei den Männern, „noch eine Herausforderung zu haben“ und das „Gefühl, gebraucht zu werden“ an vierter bzw. fünfter Stelle. Im Laufe der Erwerbstätigkeit im Rentenalter scheinen sich die Motive zu verschieben. So wechseln die am häufigsten genannten Motive in der Befragungswelle des Jahres 2019 zwischen Männern und Frauen, die finanziellen Motive finden sich auf den Rängen 2 bzw. 3. Frauen im Rentenalter in bezahlter Arbeit nennen mehrheitlich keinen konkreten Zeitplan zur Beendigung der Erwerbstätigkeit. Mergenthaler et al. (2020) vermuten, diese Ergebnisse könnten auf ungleiche Handlungsoptionen zwischen Männern und Frauen am Arbeitsmarkt hinweisen: Während Männer offenbar häufiger einen eigenen Entscheidungsspielraum hinsichtlich der Beendigung der Erwerbstätigkeit wahrnehmen, berichten Frauen davon nicht so häufig. Möglicherweise sind Frauen häufiger auf ein zusätzliches Einkommen aus Erwerbstätigkeit im Rentenalter angewiesen. Zudem verschlechtert sich bei Frauen, deren Ehepartner verstorben ist, die finanzielle Lage oftmals deutlich stärker. Eine alternative Erklärung könnte sich auf die Unterschiede in der verbleibenden Lebenszeit von Frauen und Männern beziehen. Im gleichen Lebensalter ist diese für Frauen im Mittel mehrere Jahre länger als bei Männern. Mit einer längeren verbleibenden Lebenszeit liegt eine Beendigung der Erwerbstätigkeit weiter in der Zukunft. Möglicherweise planen Frauen deshalb den Erwerbsausstieg nicht so häufig zu einem konkreten Zeitpunkt, sondern geben eher externe Bedingungen wie den Arbeitgeber oder die Gesundheit als Gründe an. 


\section{J. Deller und L. Naegele}

Subsumierend lässt sich festhalten, dass das Phänomen „Erwerbstätigkeit bzw. Weiterarbeit im Ruhestand" nicht nur zunehmend prospektiv in den Köpfen älterer Arbeitnehmerinnen und Arbeitnehmern verankert zu sein scheint, sondern inzwischen vielerorts bereits den individuellen, aber auch den betrieblichen Realitäten entspricht. Jedoch ist zu vermuten, dass die skizzierten vielfältigen Motivlagen, die individuellen Ausgestaltungsvorstellungen und der Wunsch nach Karriereentwicklung, auch im Ruhestand, in der betrieblichen Praxis oftmals auf tradierte Organisationsstrukturen und wenig alterssensible Rahmenbedingungen trifft. Eine These, die bereits von den Teilnehmerinnen und Teilnehmer der zu Beginn dieses Beitrags angesprochenen Konferenz im Jahr 1999 diskutiert wurde. Die nicht unerheblichen betrieblichen Herausforderungen, die sich hieraus ergeben, betreffen eine Vielzahl organisationaler Handlungsfelder und sollten nicht nur mit Blick auf die zunehmende Zahl der erwerbstätigen Rentnerinnen und Rentner, sondern auch mit Blick auf die generelle demografische Alterung von Belegschaften, von Betrieben adressiert werden. Der im Folgenden vorgestellte Later Life Work Index (LLWI) stellt ein beispielhaftes Instrument dar, diese Herausforderungen zu systematisieren und praxisnah Handlungsoptionen auf der betrieblichen Ebene aufzuzeigen.

\section{Die organisationale Perspektive alternsgerechter Arbeit-Later Life Work Index ${ }^{2}$}

\subsection{Erwerbsarbeit im Alter - organisationale Perspektiven}

Organisationskulturen und betriebliche Rahmenbedingungen beeinflussen die Leistungsfähigkeit und -bereitschaft im späteren Erwerbsleben und damit auch die von Personen im Rentenalter. So zeigen Studien, dass das oftmals angenommene Sinken der individuellen Produktivität im Alter (Ng und Feldmann 2008) durch den gezielten Einsatz spezifischer organisationaler Praktiken der alternsgerechten Personalentwicklung nicht nur abgefedert, sondern unter bestimmten Bedingungen sogar über das $\mathrm{Ni}$ veau von mittleren Altersgruppen hinaus gesteigert werden kann (Van Dalen, Henkens, Schippers 2010). Diesen organisatorischen Praktiken sind zuzuordnen - um hier exemplarisch nur einige wenige zu nennen - die ergonomische Neugestaltung von Arbeitsplätzen, Gesundheitsförderung, Aus- und Weiterbildung sowie Kompetenzentwicklung für ältere Beschäftigte, aber auch weniger direkte Interventionen wie die Verbesserung des Organisationsklimas, die Reduzierung von altersdiskriminierendem Verhalten und die Entwicklung eines alterssensiblen Führungsstils (Frerichs, 
Lindley, Aleksandrowicz, Baldauf und Galloway 2012 sowie Naegele, De Tavernier und Hess 2018). Bezogen auf die Gruppe der erwerbstätigen Rentnerinnen und Rentner weisen Naegele und Hess (2018) darüber hinaus auf die Notwendigkeit hin, tradierte organisationale bzw. betriebliche Karrieremodelle den Bedürfnissen und Wünschen dieser Beschäftigtengruppe anzupassen. Schaffen Unternehmen es, diese Herausforderungen anzunehmen und entsprechende Maßnahmen umzusetzen, wirkt sich dies nachweislich positiv auf die organisationalen Ergebnisse wie Leistung, Gesundheit und Motivation aus und führt zu einer verbesserten Beschäftigungsfähigkeit älterer Arbeitnehmerinnen und Arbeitnehmer aus organisationaler Sicht. Eine Möglichkeit, geeignete Rahmenbedingungen zu identifizieren und Maßnahmen einzuleiten, bietet hierbei der Later Life Workplace Index (LLWI).

\subsection{Förderliche betriebliche Rabmenbedingungen: Der Later-Life-Workplace- Index zeigt Handlungsoptionen auf}

Der Later-Life-Workplace-Index (LLWI) ist ein diagnostisches Instrument zur Einschätzung betrieblicher Praktiken und Arbeitsbedingungen im demografischen Wandel. Der Index fasst die für die Beschäftigung älterer Erwerbstätiger relevanten betrieblichen Praktiken und Arbeitsbedingungen zusammen und macht diese messbar (Wöhrmann, Dellerund Pundt 2018). Mittels Fragebögen ermöglicht der Index Organisationen, die eigenen Stärken und Schwächen hinsichtlich des Umgangs mit älteren Beschäftigten zu identifizieren, sich im Rahmen eines anonymen Benchmarks mit anderen Organisationen zu vergleichen und Handlungsoptionen abzuleiten. Deller et al. (im Druck) entwickelten den LLWI mit drei Hauptzielen: Erstens soll der Index ein ganzheitliches Bild der wichtigsten betrieblichen Praktiken und Rahmenbedingungen vermitteln. Der LLWI misst mit allen relevanten Dimensionen die wichtigsten Aspekte ganzheitlich und ermöglicht damit den Vergleich zwischen verschiedenen Praktiken und betrieblichen Rahmenbedingungen. Zweitens soll die Einschätzung mit möglichst geringem Aufwand durchzuführen sein, um im Tagesgeschäft betrieblicher Personalarbeit anwendbar zu sein. Und drittens soll die Operationalisierung so spezifisch sein, dass Verbesserungspotenziale identifiziert und Maßnahmen und Interventionsprogramme abgeleitet werden können.

Die im LLWI enthaltenen organisatorischen Praktiken und Arbeitsbedingungen wurden empirisch aus Experteninterviews mit älteren Beschäftigten, erwerbstätigen Rentnerinnen und Rentnern, Vertreterinnen und Vertretern der Wirtschaft, der Politik und der Wissenschaft aus den Berei- 
chen Personalwirtschaft, Betriebswirtschaft, Psychologie und Gerontologie erarbeitet. Durch den multidisziplinären Hintergrund der Expertinnen und Experten wurde sichergestellt, dass die identifizierten Aspekte die betrieblichen Stellschrauben umfassend abbilden (Wöhrmann et al. 2018). Die identifizierten Aspekte wurden abschließend durch eine Integration mit den Praktiken des Age Smart Employer Award validiert (Wilckens, Wöhrmann, Adams, Deller und Finkelstein 2020). Der LLWI besteht aus neun Dimensionen, wie in Abbildung 1 dargestellt. Die beiden Dimensionen Organisationsklima und Führung ergänzen sieben weitere spezifische Praktiken und Arbeitsbedingungen. Jede Dimension wird durch zwei bis vier Indikatoren näher erläutert. ${ }^{3}$

Abbildung 1. Dimensionen des LLWI und ibre Indikatoren (Wilckens et al. 2019)

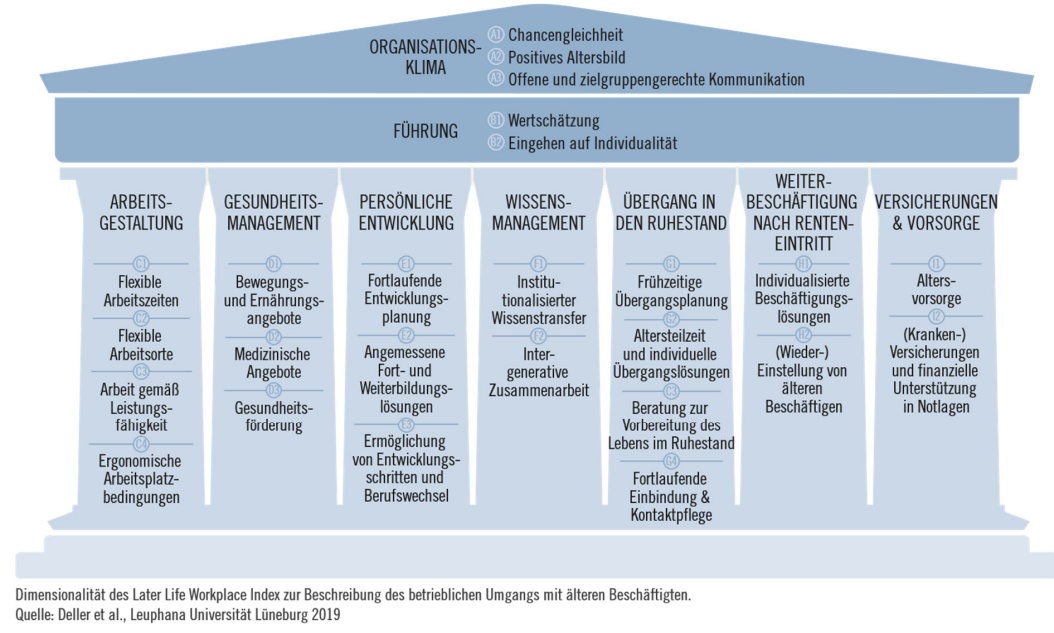

Die Einschätzung von Organisationen entlang des LLWI erfolgt mittels Fragebögen, die alle Dimensionen und Indikatoren messen und von einer kleinen, möglichst breit gestreuten Stichprobe aus älteren Beschäftigten, Führungskräften sowie einer Vertreterin oder einem Vertreter der Personalabteilung innerhalb jeder Organisation beantwortet werden. Durch die Befragung von sowohl Vertreterinnen und Vertretern des Personalwesens als auch der Führungskräfte und der älteren Beschäftigten erfasst der Index nicht nur, welche Bedingungen prinzipiell vorherrschen sollten, sondern auch, wie diese durch die älteren Beschäftigten selbst wahrgenommen wer- 
den. Jeder Indikator wird mit zwei bis vier Fragen erfasst. Der Aufwand der Befragung ist dadurch überschaubar, während gleichzeitig eine zuverlässige Messung gewährleistet ist. Allen teilnehmenden Organisationen werden Ergebnisdarstellungen zur Verfügung gestellt. Die Rückmeldung erfolgt auf Ebene der Dimensionen, Indikatoren und Einzelfragen. So erhalten Organisationen zunächst einen schnell erfassbaren Überblick über die Auswertung und können dann einzelne Bereiche vertiefen, um gezielt Handlungsoptionen abzuleiten. Dabei werden die Ergebnisse stets mit den Antworten ähnlicher Organisationen verglichen. Eine beispielhafte Ergebnisdarstellung für die Einzelfragen eines Indikators der Dimension Übergang in den Ruhestand ist in Abbildung 2 dargestellt.

Die Antworten der teilnehmenden Einzelpersonen in der Organisation werden dafür jeweils zu einem Mittelwert zusammengefasst. Dieser ist in der Ergebnispräsentation als türkiser Streifen markiert. Die mittlere Hälfte der Antworten der Vergleichsstichprobe ist als hellblauer Balken dargestellt. Das bedeutet, dass bei insgesamt 100 nach Größe sortierten Antworten die 25. Antwort am linken und die 75. Antwort am rechten Rand des Balkens liegt. Die graue Raute markiert den Mittelwert der Vergleichsgruppe. Am rechten Rand der Abbildung befindet sich eine Ampel, welche die Abweichung des Mittelwerts der Organisation zu dem der Vergleichsgruppe wiedergibt. Dabei wird die Abweichung relativ zur Standardabweichung der Vergleichsgruppe gemessen. Eine rote Ampel bedeutet, dass die Organisation eine Standardabweichung schlechter als der Mittelwert der Vergleichsgruppe abschneidet. Bei einer grünen hingegen liegt der Messwert der Organisation eine Standardabweichung besser als der Mittelwert der Vergleichsgruppe. 
Abbildung 2. Ausschnitt aus der Ergebnisdarstellung für die Dimension Übergang in den Ruhestand (Beispiel)

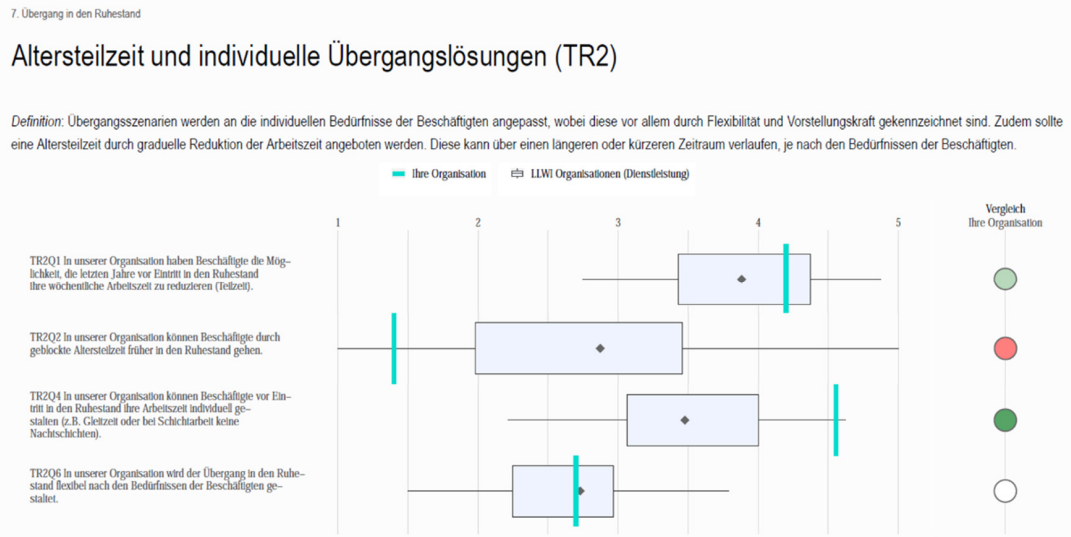

Die aus dem Projekt gewonnenen Daten dienen zum einen als Benchmark-Datenbank, sodass Organisationen ihre LLWI-Ergebnisse mit denen ähnlicher Organisationen anonym vergleichen können und auf Basis eines Vergleichs Transparenz über die eigenen Stärken und Schwächen erhalten. Ein besseres Verständnis und Bewusstsein für die Voraussetzungen und Möglichkeiten eines verlängerten Arbeitslebens auf Unternehmensebene, die Möglichkeiten für eine fundierte Diskussion von Stärken und Verbesserungspotenzialen sowie die abzuleitenden Maßnahmen kommen sowohl dem Unternehmen in Form gesteigerter Gesundheit, Leistung und Motivation als auch den älteren Beschäftigten zugute. Deller et al. (im Druck) entwickeln daher parallel zum Messinstrument LLWI einen Methodenkasten mit 48 betrieblichen Maßnahmen als konkrete Handlungsansätze entlang der LLWI-Dimensionen.

Der LLWI stellt somit ein wissenschaftlich entwickeltes diagnostisches Instrument dar, das betriebliche Rahmenbedingungen im demografischen Wandel ganzheitlich betrachtet und die Organisationen dabei unterstützt, ältere Beschäftigte langfristig erfolgreich zu beschäftigen. Der LLWI trägt somit zur Verbesserung der organisatorischen Rahmenbedingungen für einen erfolgreichen Umgang mit alternden Belegschaften und damit zur gesellschaftlichen Bewältigung des demografischen Wandels bei. Seine Inhalte fließen aktuell auch in die Gestaltung einer nationalen DIN- sowie einer internationalen ISO-Norm zur Gestaltung von Arbeit angesichts alternder Belegschaften ein. 


\subsection{Beispiele: Persönliche Entwicklung und Übergang in den Ruhestand}

An dieser Stelle sollen beispielhaft die im LLWI adressierten Dimensionen Persönliche Entwicklung und Übergang in den Ruhestand mit ihren jeweiligen Indikatoren und daraus abgeleiteten betrieblichen Handlungsansätzen dargestellt werden. Beide Dimensionen sind dabei in besonderer Weise bedeutsam, es soll alternden Belegschaften bzw. der wachsenden Anzahl erwerbstätiger Rentnerinnen und Rentner ein erfolgreiches „Durchaltern“ im Erwerbsleben ermöglicht werden. Sie nehmen dabei zum einen die individuelle Entwicklungsperspektive von Beschäftigten in den Blick, adressieren die Notwendigkeit, den Übergang in den Ruhestand adäquat zu planen und nehmen hier insbesondere auch die Betriebe in die Verantwortung.

Die erste Dimension Persönliche Entwicklung umfasst die Unterstützung der Beschäftigten in ihrer beruflichen und persönlichen Entwicklung über den gesamten Erwerbsverlauf hinweg. Die Dimension enthält folgende Indikatoren:

- Fortlaufende Entwicklungsplanung: Es erfolgt unabhängig von Alter und Karrierestufe eine kontinuierliche Planung der individuellen Entwicklungsperspektiven für alle Beschäftigten, z.B. in Form von Entwicklungsgesprächen oder Workshops zur beruflichen Standortbestimmung, um die eigenen Fähigkeiten, Kompetenzen und Ziele zu reflektieren.

- Angemessene Fort- und Weiterbildungslösungen: Es werden Weiterbildungs- und Fortbildungsmaßnahmen angeboten, die individuelle Lebens-, Berufs- und Lernerfahrung sowie organisationale Ziele berücksichtigen. Außerdem sollten Trainingsmethoden und -inhalte der jeweiligen Zielgruppe entsprechend eingesetzt werden. Beispiele für angemessene Fort- und Weiterbildungslösungen können Seminare, Workshops und Konferenzen, Trainings für neue Technologien und Praktika in anderen Bereichen für alle Altersgruppen sein. Ermöglicht werden können diese durch die Erstattung von entstehenden Kosten.

- Ermöglichung von Entwicklungsschritten und Arbeitsplatzwechsel: Es wird eine Modifikation der aktuellen Tätigkeit oder des aktuellen Arbeitsplatzes ermöglicht, welcher den spezifischen Kompetenzen und Wünschen der Beschäftigten entgegenkommt, z.B. durch Aufgabenerweiterung, die Einbindung in Projekte oder einen horizontalen sowie vertikalen beruflichen Richtungswechsel, der auch eine neue Berufsausbildung oder eine neue Tätigkeit in einer anderen Abteilung bedeuten kann. 
Die Dimension Übergang in den Ruhestand beinhaltet die notwendigen Planungen, Vereinbarungen und Vorbereitungen für alle Beschäftigten, die kurz vor dem Ruhestand stehen oder in diesen eintreten. Die Indikatoren lauten:

- Frühzeitige Übergangsplanung: Mit den Beschäftigten wird frühzeitig über deren persönliche Planung und mögliche Übergangsszenarien und Nachfolgelösungen gesprochen, um so für diese individuellen Lösungen zu finden. Dies geschieht z.B. im Rahmen der jährlichen Mitarbeitergespräche.

- Altersteilzeit und individuelle Übergangslösungen: Übergangsszenarien werden an die individuellen Bedürfnisse der Beschäftigten angepasst, wobei diese vor allem durch Flexibilität und Vorstellungskraft gekennzeichnet sind. Zudem sollte eine Altersteilzeit durch graduelle Reduktion der Arbeitszeit angeboten werden. Diese kann über einen längeren oder kürzeren Zeitraum verlaufen, je nach den Bedürfnissen der Beschäftigten.

- Beratung zur Vorbereitung des Lebens im Ruhestand: Organisationen sollten ihre Beschäftigten durch ein Beratungsangebot darin unterstützen, sich mental auf die Veränderungen durch den Ruhestand einzustellen und die Ruhestandsphase aktiv zu gestalten. Beispielsweise kann die individuelle Vorbereitung durch einen strukturierten Ansatz, der persönliche Erwartungen und Pläne reflektiert, gefördert werden. Außerdem können die Beschäftigten darin unterstützt werden, alternative Beschäftigungsmöglichkeiten aufzubauen.

- Fortlaufende Einbindung und Kontaktpflege: Es werden Maßnahmen ergriffen, um den Kontakt zu Beschäftigten, die sich im Übergang in die Ruhestandsphase befinden, zu halten und ihnen zu vermitteln, dass sie weiterhin Teil der Organisation sind, z.B. durch eine aktive Beziehungspflege in Form eines Alumni Netzwerks, die Einladung zu Veranstaltungen oder durch das Angebot zur Übernahme freiwilliger Aufgaben in der Organisation.

\section{Fazit: Diskussion und Ausblick}

Das Thema Arbeit im Ruhestand, so hat der Rückblick zu Beginn des Kapitels gezeigt, beschäftigt die betriebliche Praxis und Wissenschaft seit über zwei Dekaden. Während im Jahr 1999 die betriebliche Argumentation primär die Praxis der Frühverrentung bzw. „Rente mit 60“ zu priorisieren schien, wiesen die Teilnehmerinnen und Teilnehmer der Konferenz 
vor dem Hintergrund zukünftiger Engpässe im Erwerbspersonenpotenzial bereits damals auf die Notwendigkeit hin, Arbeitswelten neu zu gestalten (Naegele 2000). Solle das Ziel von verlängerten Erwerbskarrieren erreicht werden, bedürfte es konzeptioneller Weiterentwicklungen mit Blick auf alternde Belegschaften, die damals noch nicht in den Betrieben angekommen zu sein schienen. Inzwischen sind - zumindest der aktuellen Datenlage nach - ältere Beschäftigte „angekommen“ in den betrieblichen Realitäten und unter ihnen besonders die stetig wachsende Gruppe der erwerbstätigen Rentnerinnen und Rentner (Mergenthaler et al. 2020). Über Letztere wissen wir darüber hinaus aktuell deutlich mehr, beispielsweise über deren Motivlagen, Ausgestaltungswünsche und Karrierevorstellungen bezogen auf eine Erwerbstätigkeit im Ruhestand. Hier zeigt sich, dass nicht allein monetäre Gründe ausschlaggebend zu sein scheinen (Büsch et al. 2015 sowie Fasbender et al. 2015), bei der Entscheidung im Ruhestand zu arbeiten. Vielmehr zeigen sich Motivlagen, die den Wunsch nach sozialer Einbindung aber auch individueller Weiterqualifizierung nahelegen. Gleichzeitig wünschen sich erwerbstätige Rentnerinnen und Rentner mehr Flexibilität bezogen auf Arbeitsort und -zeit sowie Karriereentwicklungsoptionen (Naegele und Hess 2018).

Wechselt man jedoch die Perspektive auf die betriebliche Ebene, d.h. dahin, wo ein Großteil der Personen, die aktuell einer Beschäftigung im Ruhestand nachgehen, zu finden sind, findet man dort eine Dominanz geringfügiger Beschäftigungsverhältnisse, nicht unerhebliche Geschlechterund Bildungsunterschiede, einen starken Gesundheitsbias und oftmals wenig Qualifizierungs- und Karriereentwicklungsangebote für Rentnerinnen und Rentner (Naegele 2020). Dies muss nicht unbedingt konträr zu den Wünschen erwerbstätiger Rentnerinnen und Rentner stehen, ist jedoch als problematisch zu werten, sollten sich hiermit soziale Ungleichheiten verhärten. Die oftmals heute im Ruhestand vorherrschenden Beschäftigungsverhältnisse (z.B. Mini-Jobs oder befristete Projekt- bzw. Beratungsverträge) können vielleicht einem Teil der erwerbstätigen Rentnerinnen und Rentner die gewünschte Flexibilität und Selbstbestimmung bieten, gleichermaßen für andere Personengruppen in prekären Lebenslagen - die angewiesen sind auf einen Zuerwerb im Ruhestand - eine berufliche Einbahnstraße bedeuten. Auch eine berufliche Neuorientierung im Ruhestand (z.B. bei einem neuen Arbeitgeber mit anderen Tätigkeitsschwerpunkten), wie es sich in etwa ein Viertel der TOP-Befragten wünschen (Naegele und Hess 2018), ist vor den skizzierten Hintergrundbedingungen oftmals nur schwer möglich. Fraglich ist auch, inwieweit im Ruhestand überhaupt von alterssensiblen Arbeitsumwelten ausgegangen werden kann, wenn diese vielerorts kaum für ältere Erwerbstätige vor dem Ruhe- 


\section{J. Deller und L. Naegele}

stand umgesetzt zu werden scheinen. Vor diesem Hintergrund ist zu befürchten, dass bereits bestehende Chancenungleichheiten im höheren Erwerbsalter in den Ruhestand übertragen werden, sich dort verfestigen und neue soziale Ungleichheiten produzieren. Dies gilt in besonderer Weise, wenn sich - so die Vermutung hier - zeigen sollte, dass diese Form der Erwerbstätigkeit meist eh nur gesundheitlich nicht eingeschränkten und hochqualifizierten Rentnerinnen und Rentnern offensteht.

Der Later-Life-Workplace-Index (LLWI) - ganz in der Tradition der im Jahr 1999 bereits geforderten Neuerungen - stellt an dieser Stelle eine wichtige konzeptionelle Weiterentwicklung für die Mesoebene, die betriebliche Realität, dar. Ist es das Ziel auf betrieblicher Ebene, die Grundlagen für die systematische Umsetzung von (erfolgreicher) Weiterarbeit im Ruhestand zu schaffen und damit vielleicht auch bestehende soziale Ungleichheiten abzubauen, benötigt es den prospektiven (vorbereitenden) Charakter der im LLWI vorgeschlagenen Verbesserungs- und Interventionsprogramme. Gleichermaßen muss ein entsprechendes Instrument möglichst niedrigschwellig angelegt sein, damit es von den betrieblichen Akteuren auch zum Einsatz gebracht wird. Mit dem LLWI steht nun ein erster gut validierter Ansatz für die Betriebe zur Verfügung, dessen Praxisbewährung allerdings noch aussteht. Vor diesem Hintergrund sind insbesondere Interventionsstudien notwendig, die auch die Praxiswirksamkeit validieren. Auch ist noch unklar, welche Wirkung die angesprochenen Normen von DIN und ISO, in die die Inhalte des LLWI eingeflossen sind, in der Praxis entfalten werden. Es bleibt zu hoffen, dass diese eine höhere Durchschlagkraft entwickeln, als es die Forderungen der Teilnehmerinnen und Teilnehmer der Konferenz aus dem Jahr 1999 getan haben.

An dieser Stelle ist neben der Praxis aber auch die Wissenschaft gefragt, um bestehende offene Fragen zu adressieren. Wünschenswert wären hier beispielsweise Mehrebenenbetrachtungen des Zusammenspiels von Makro- (z.B. politische und gesellschaftliche Randbedingungen etc.), Meso(organisationale, institutionelle und private außerberufliche Stellgrößen etc.) und Mikrofaktoren (Motivationsdimensionen, Persönlichkeitsmerkmale, Zielorientierungen etc.). Dies erfordert transdisziplinäre Forschungsgruppen, entsprechend gestaltete innovative Datenerhebungen und Weiterentwicklungen in der Modellierung (vgl. Büsch et al. 2015). Auch ist von Interesse, inwieweit die „neue“ Beschäftigtengruppe der erwerbstätigen Rentnerinnen und Rentner tradierte Handlungsweisen und Karrierewege in der betrieblichen Praxis verändern und wie sie sich insgesamt in existierende Betriebsgefüge eingliedern. Darüber hinaus gilt es, ein Augenmerk auf die Gefahr der Verfestigung sozialer Ungleichheiten, die im Zuge des Ausbaus von Erwerbstätigkeit im Rentenalter entstehen können, zu 
werfen. Dazu müssen Wege und Maßnahmen entwickelt und validiert werden, welche die individuellen, organisationalen und gesellschaftlichen Chancen - die in verlängerten Erwerbskarrieren stecken - fördern, ohne dabei bestimmte (prekäre) Beschäftigungsgruppen zu exkludieren.

\section{Literatur}

Auer, P. (2000): Altern: Herausforderungen und Antworten. In: C. von Rothkirch (Hrsg.), Altern und Arbeit: Herausforderung für Wirtschaft und Gesellschaft, S. 345-346, Berlin: Ed. Sigma.

Ax, C. (2000): Handwerk: Zukunft in einer alternden Gesellschaft - Einblicke und Ausblicke. In: C. von Rothkirch (Hrsg.), Altern und Arbeit: Herausforderung für Wirtschaft und Gesellschaft, S. 148-166, Berlin: Ed. Sigma.

Behrens, J. (2000): Was Demographie mit Kinder kriegen zu tun hat und was uns vorzeitig alt aussehen lässt - Illusionen im Trendmodell der Erwerbszeit. In C. von Rothkirch (Hrsg.), Altern und Arbeit: Herausforderung für Wirtschaft und Gesellschaft, S. 228-259, Berlin: Ed. Sigma.

Behrens, J., Morschhäuser, M., Viebrok, H. und Zimmermann, E. (1999): Länger erwerbstätig - aber wie? Opladen: Westdeutscher Verlag.

Böhle, F. (2000): Alter und Arbeit - Erwerbsarbeit zwischen Markt und Staat als neue Herausforderung für die Arbeitsmarkt- und Sozialpolitik. In: C. von Rothkirch (Hrsg.), Altern und Arbeit: Herausforderung für Wirtschaft und Gesellschaft, S. 310-338, Berlin: Ed. Sigma.

Büsch, V., Zohr, K., Brusch, M., Deller, J., Schermuly, C. C., Stamov-Roßnagel, C. und Wöhrmann, A. M. (2015): Wer möchte im Ruhestand weiterarbeiten? Muster von Weiterbeschäftigungsneigungen bei 55- bis 70-Jährigen. In: N. Schneider, A. Mergenthaler, U. M. Staudinger und I. Sackreuther (Hrsg.), Mittendrin? Lebenspläne und Potenziale älterer Menschen beim Übergang in den Ruhestand, S. 181-193, Opladen: Budrich. Verfügbar unter: https://www.bib.bund.de /Publikation/2015/pdf/Mittendrin-Lebensplaene-und-Potenziale-aelterer-Mensch en-beim-Uebergang-in-den-Ruhestand.pdf?_blob=publicationFile\&v=1.

Deller, J., Kern, S., Hausmann, E. und Diederichs, Y. (2008): Personalmanagement im demografischen Wandel: Ein Handbuch für den Veränderungsprozess. Berlin: Springer.

Deller, J., Liedtke, P. M. und Maxin, L. M. (2009): Old-age security and Silver Workers: An empirical investigation identifies challenges for companies, insurers, and society. Geneva Papers on Risk and Insurance, 34, S. 137-157. DOI:10.1057/gpp.2008.44.

Deller, J. und Maxin, L. M. (2009): Berufliche Aktivität von Ruheständlern. Zeitschrift für Gerontologie und Geriatrie, 42, S. 305-310. DOI:10.1007/s00391-0090047-3. 
Deller, J. und Pundt, L. M. (2015): Wie Firmen mit Silver Work dem wachsenden Fachkräftemangel begegnen können - Wege aus der Beschäftigungskrise. In: Goinger Kreis (Hrsg.), Zukunft Personal Beschäftigung - Zwischen Praxis und Innovation, Unternehmen und Gesellschaft, S. 117-126, Wiesbaden: Springer Gabler. DOI:10.1007/978-3-658-09196-5.

Deller, J., Wöhrmann, A. M., Wilckens, M. und Finsel, J. (im Druck): Later Life Workplace Index - Demographiemanagement für Organisationen. In: K.-H. Schwuchow und J. Gutmann (Hrsg.), HR-Trends 2021. Freiburg: Haufe.

Fasbender, U., Deller, J., Zohr, K., Büsch, V., Schermuly, C. C. und Mergenthaler, A. (2015): Absicht zur Erwerbstätigkeit im (zukünftigen) Ruhestand. In: N. Schneider, A. Mergenthaler, U. M. Staudinger und I. Sackreuther (Hrsg.), Mittendrin? Lebenspläne und Potenziale älterer Menschen beim Übergang in den Ruhestand, S. 121-137, Opladen: Budrich.

Frerichs, F., Lindley, R., Aleksandrowicz, P., Baldauf, B. und Galloway, S. (2012): Active ageing in organisations: A case study approach. International Journal of Manpower, 33(6), S. 666-684.

Finsel, J., Wilckens, M., Wöhrmann, A. M. und Deller, J. (under review): Later Life Workplace Index - Ein Instrument zur Unterstützung betrieblicher Beschäftigungspraktiken. In: G. Naegele und M. Heß (Hrsg.), Alte und neue soziale Ungleichheiten beim Rentenübergang - Ergebnisse des EXTEND Projektes. Wiesbaden: Springer VS.

Griffin, B. und Hesketh, B. (2008): Post-Retirement work: The individual determinants of paid and volunteer work. Journal of Occupational and Organizational Psychology, 81(1), S. 101-121. DOI:10.1348/096317907X202518.

Hellferich, B. (2000): Die Frage des Alterns in der Europapolitik. In: C. von Rothkirch (Hrsg.), Altern und Arbeit: Herausforderung für Wirtschaft und Gesellschaft, S. 19-22, Berlin: Ed. Sigma.

Hess, M. und Naegele, L. (im Erscheinen): Aktivitäten bei fortgesetzter Arbeit. In: H. Jürgens, J. Siegrist, und M. Stiehler (Hrsg.), Männer und ihre Gesundheit vor und nach der Rente. Buchreihe: Forschung Psychosozial. Gießen: Psychosozial Verlag.

Kador, F.-J. (2000): Zusammenfassung. In: C. von Rothkirch (Hrsg.), Altern und Arbeit: Herausforderung für Wirtschaft und Gesellschaft, S. 347-348, Berlin: Ed. Sigma.

Kruse, A. (2000): Psychologische Beiträge zur Leistungsfähigkeit im mittleren und höheren Erwachsenenalter - eine ressourcenorientierte Perspektive. In: C. von Rothkirch (Hrsg.), Altern und Arbeit: Herausforderung für Wirtschaft und Gesellschaft, S. 72-87, Berlin: Ed. Sigma.

Maxin, L. und Deller, J. (2010): Beschäftigung statt Ruhestand: Individuelles Erleben von Silver Work. Zeitschrift für Bevölkerungswissenschaft, 35, S. 767-800. DOI:10.4232/10.CPoS-2010-18de. 
Mergenthaler, A., Konzelmann, L., Cihlar, V., Micheel, F. und Schneider, N. F. (2020): Vom Ruhestand zu (Un)Ruheständen. Ergebnisse der Studie „Transitions and Old Age Potential (TOP) von 2013-2019“. Wiesbaden: Bundesinstitut für Bevölkerungsforschung. Verfügbar unter: https://www.bib.bund.de/Publikat ion/2020/pdf/Vom-Ruhestand-zu-Un-Ruhestaenden.pdf?_blob=publicationFile $\& v=8$.

Morschhäuser, M. (2000): Personalentwicklung oder Personalaustausch? Perspektiven alternsbezogener Personalplanung. In: C. von Rothkirch (Hrsg.), Altern und Arbeit: Herausforderung für Wirtschaft und Gesellschaft, S. 282-293, Berlin: Ed. Sigma.

Naegele, G. (2000a): Ausblick aus Sicht der Wissenschaft. In: C. von Rothkirch (Hrsg.), Altern und Arbeit: Herausforderung für Wirtschaft und Gesellschaft, S. 436-442, Berlin: Ed. Sigma.

Naegele, G. (2000b): Trends und Herausforderungen - Einführung in den Kongress „Altern und Arbeit“. In: C. von Rothkirch (Hrsg.), Altern und Arbeit: Herausforderung für Wirtschaft und Gesellschaft, S. 23-26, Berlin: Ed. Sigma.

Naegele, L., De Tavernier, W. und Hess, M. (2018): Work environment and the origin of ageism. In: L. Ayalon und C. Tesch-Römer (Hrsg.), Contemporary Perspectives on Ageism. International Perspectives on Aging, Bd. 19. Cham: Springer.

Naegele, L., Stiemke, P., Hess, M. und Mäcken, J. (2020): (Wie) wollen wir im Rentenalter arbeiten? Eine Untersuchung zu den Beschäftigungsvorstellungen zukünftig erwerbstätiger Rentnerinnen und Rentner in Deutschland. In: U. Fachinger und Fr. Frerichs (Hrsg.), Selbstständige Erwerbstätigkeit und Erwerbskarrieren in späteren Lebensjahren - Potentiale, Risiken und Wechselwirkungen, S. 211-238, Vechtaer Beiträge zur Gerontologie. Springer.

Naegele, L. und Hess, M. (2018): Karrieren nach der Rente: Karriere- und Arbeitsvorstellungen von arbeitenden Rentner*innen. Gruppe. Interaktion. Organisation. Zeitschrift für Angewandte Organisationspsychologie (GIO), 48(1), S. 58-68.

Naegele, L. (2020): Betriebliches Kompetenzmanagement älterer Arbeitnehmer*innen - Eine betriebssoziologische Analyse im Handwerk. Vechtaer Beiträge zur Gerontologie. Springer.

Ng, T. W. H. und Feldman, D. C. (2008): The relationship of age to ten dimensions of job performance. The Journal of Applied Psychology, 93(2), S. 392-423.

o.V. (2000): Podiumsdiskussion. In: C. von Rothkirch (Hrsg.), Altern und Arbeit: Herausforderung für Wirtschaft und Gesellschaft, S. 45-69, Berlin: Ed. Sigma.

Rothkirch, C. von (2000) (Hrsg.): Altern und Arbeit: Herausforderung für Wirtschaft und Gesellschaft. Berlin: Ed. Sigma.

Spieker, H. (2000): Erfahrungen mit Personalplanung für den Lohnbereich. Praxisbericht von einem betrieblichen Arbeitsmarkt. In: C. von Rothkirch (Hrsg.), Altern und Arbeit: Herausforderung für Wirtschaft und Gesellschaft, S. 294-303, Berlin: Ed. Sigma. 
Van Dalen, H. P., Henkens, K., Schippers, J. (2010): Productivity of older workers: Perceptions of employers and employees. Population and Development Review, 36(2), S. 309-333.

Wilckens, M. R., Finsel, J., Wöhrmann, A. M. und Deller, J. (2019): Alternde Belegschaften: Der Later Life Work Index adressiert betriebliche Herausforderungen und zeigt individuell Lösungsansätze auf. In Goinger Kreis e.V. (Hrsg.), Grenzüberschreitungen zwischen Unternehmen und Gesellschaft - Herausforderungen im System Arbeit gemeinsam bewältigen, S. 359-384, Bad Homburg v.d.H.: VAS-Verlag für Akademische Schriften.

Wilckens, M. R., Wöhrmann, A. M., Adams, C., Deller, J. und Finkelstein, R. (2020): Integrating the German and US perspective on organizational practices for later-life work: The Later Life Work Index. In: S. Czaja, J. Sharit, J. James und J. Grosch (Eds.), Current and emerging trends in aging and work, S. 59-79, Cham: Springer. DOI:10.1007/978-3-030-24135-3.

Wilckens, M. R., Wöhrmann, A. M., Deller, J. und Wang, M. (in print). Organizational practices for the aging workforce: Development and validation of the Later Life Workplace Index (LLWI). Work, Aging and Retirement.

Wöhrmann, A. M., Deller, J. und Pundt, L. (2018); Complementing AAI at the meso level - The Silver Work Index. In: A. Zaidi, S. Harper, K. Howse, G. Lamura und J. Perek-Białas (Eds.), Building evidence for active ageing policies: Active Ageing Index and its potential, S. 75-94, London: Palgrave MacMillan. DOI: 10.1007/978-981-10-6017-5.

\section{Internetlink}

www.later-life-workplace-index.org

\section{Referenzen}

1 Nicht unbeachtet bleiben sollten an dieser Stelle auch diejenigen Rentnerinnen und Rentner, die im Ruhestand einer unbezahlten z.B. ehrenamtlichen Beschäftigung nachgehen. So handelt es sich auch hier um sinnstiftende, einen gesellschaftlichen Mehrwert generierende, Beschäftigung, die jedoch in diesem Beitrag nicht weiter behandelt werden soll. Für einen vergleichenden Blick auf bezahlte und unbezahlte Tätigkeiten im Rentenalter siehe: Griffin und Hesketh (2008).

2 Dieses Teilkapitel nutzt Material aus Deller, Wöhrmann, Wilckens und Finsel (im Druck).

3 Eine Definition der verschiedenen Dimensionen und Indikatoren findet sich auf der Projektwebsite der Leuphana Universität Lüneburg: https://www.leuphana.d e/en/portals/later-life-workplace-index.html. 